\title{
Immunohistochemical expression of SIRT1 in oral squamous cell carcinoma and its relationship with clinical-pathological factors
}

\author{
Maryam Seyedmajidi, Shima Nafarzadeh, Arezoo Rayani,, Dariush Moslemi, ${ }^{\text {A }}$ Ali Bijani, \\ and Majid Sharbatdaran ${ }^{\dagger}$
}

\author{
aDental Materials Research Center, School of Dentistry, Babol University of Medical Sciences, Babol, Iran. \\ bDepartment of Oral and Maxillofacial Pathology, School of Dentistry, Babol University of Medical Sciences, Babol, Iran. \\ ‘Department of Oral and Maxillofacial Pathology, School of Dentistry, Mazandaran University of Medical Sciences, Sari, Iran. \\ dDepartment of Radiation Oncology, Babol University of Medical Sciences, Babol, Iran \\ eNon-Communicable Pediatrics Diseases Research Center, Babol University of Medical Sciences, Babol, Iran. \\ fDepartment of Pathology, Babol University of Medical Sciences, Babol, Iran. \\ Correspondence to Arezoo Rayani (email: arezoo.rayyani@gmail.com). \\ (Submitted:02 August 2019 - Revised version received: 18 August 2019 - Accepted: 15 September 2019 - Published online: 26 October 2019)
}

\begin{abstract}
Objective Oral squamous cell carcinoma (OSCC) is the most common oral cancer in the world that threatens public health. There are a number of molecular markers that can be used to improve TNM staging. One of these markers is SIRT1. We aimed to investigate the expression of SIRT1 in OSCC and its relationship with clinicopathological factors such as age, sex, location of tumor, smoking, clinical stage, grade of tumor, metastasis to lymph nodes in the neck and distant metastasis.

Methods This cross-sectional study was performed on 30 samples of OSCC and 30 samples of normal oral mucosa. Required clinical data were collected from patients. After immunohistochemistry staining for SIRT1 on sections prepared with paraffin blocks, the percentage of stained cells and staining intensity of the cells were evaluated in the terms of core and cytoplasmic aspects.

Results Positive expression of SIRT1 was seen in $93.3 \%$ and $96.7 \%$ of cells in the core and cytoplasm view, respectively, which was significantly higher than normal tissue ( $P=0.021$ and 0.001 , respectively). We found no significant relationship between the intensity and percentage of core and cytoplasmic staining of squamous cells as well as clinicopathological factors.

Conclusion The overexpression of SIRT1 in OSCC samples as compared with normal mouth tissue indicates its role in carcinogenesis. However, further studies on SIRT1 may shed more light on the treatment options of OSCC.

Keywords squamous cell carcinoma, immunohistochemical staining, SIRT1
\end{abstract}

\section{Introduction}

Squamous cell carcinoma (SCC) is the most common malignancy of the oral cavity. Most samples of oral squamous cell carcinoma (OSCC) usually have no visible changes in the early stages. Therefore, early detection can be crucial in order to improve the lives of afflicted individuals and constitutes $90-95 \%$ of all oral malignancies and the fifth most common cancer worldwide. ${ }^{1-3}$ The mortality rate is about $50 \%$ and has not changed in the past 50 years despite extensive research., 4

The prevalence of this disease is on the rise in youth. The main contributing factor to OSCC in people under 40 years of age is uncertain and the main risk factors (alcohol and tobacco) is often absent in this population. ${ }^{6}$ SIRT1, also known as NAD-dependent histone deacetylase ${ }^{3}$ is an orthologous gene of SIR2 and has multiple roles. ${ }^{7}$ This protein prohibits rRNA transcription by deacetylating histone of H3K9 leading to the inhibition of apoptosis. Also, the expression of SIRT1 with deacetylated histones has an oncogenic effect and reduces the activity of tumor suppressors such as ku70, p53, and foxo protein family. ${ }^{8}$

SIRT1 is involved in DNA repair and can act as a tumor suppressor. ${ }^{9}$ Therefore, SIRT1 plays different roles in different types of cancer. Its expression is increased in stomach, breast, and prostate cancers. ${ }^{3}$ With the help of $\beta$-catenin and c-Myc, SIRT1 plays an important role in tumor progression and metastasis. ${ }^{3}$ Studies show that this protein has an oncogenic role through the inhibition of apoptosis, differentiation, proliferation, and growth. ${ }^{10}$
TNM is commonly used to predict the clinicopathological status in cancers. However, the predictive value of the staging system is not enough. There are a number of molecular markers that can be effective in improving the ability of staging system. ${ }^{11,12}$

Studies on the use of SIRT1 as a therapeutic molecule goal for OSCC are scarce. There is no comprehensive study about the relationship between the expression of SIRT1 and clinicopathological factors in patients with OSCC and most studies have been done in other parts of the body. Therefore, we aimed to investigate the expression of SIRT1 in OSCC and its relationship with clinicopathological factors such as age, sex, location of tumor, smoking, clinical stage, grade of tumor, metastasis to lymph nodes in the neck, and distant metastasis.

\section{Materials and Methods}

\section{Sampling}

In this cross-sectional study, 30 paraffin blocks of OSCC were obtained from the archives of the Department of Pathology in hospitals of Mazandaran Province, northern Iran.

Also, 30 paraffin blocks of normal oral mucosa obtained from patients who referred for crown lengthening surgery and had minimal inflammation or change in clinical and histopathological condition were studied. Records of patients diagnosed with OSCC were extracted non-randomly and after recording the patients' demographic data, the relevant blocks were extracted. 
Required information including age, sex, location of tumor, smoking, clinical tumor stage, grade of tumor, neck lymph node metastasis, and distant metastasis were obtained from their pathological reports or during contact with patients. Two cuts were prepared from the paraffin blocks. The first cut with a thickness of 4 microns was hematoxylin-eosin stained to confirm the initial diagnosis and select appropriate blocks containing enough samples. The second cut was prepared for immunohistochemical study.

\section{SIRT1 Immunohistochemistry}

About $3 \mu \mathrm{m}$ thick slice was prepared from paraffin blocks and mounted on Sailyn slide. Tissue sections were dehydrated and deparaffined.

Deparaffined samples for antigen retrieval by Lam Holder (Dako, Copenhagen, Denmark) was placed in buffer citrate 0.01 M with 2.6-6 PH and subjected to microwave and then the slides were exposed to hydrogen peroxide with for $10 \mathrm{~min}$. Next, the surface of the tissue was completely covered for 30 min with primary polyclonal antibodies of anti-SIRT1 antibody Rabbit (ART NOsc-15404, Santa Cruz, Biotechnology, Santa Cruz, CA, USA). Then, the tissue surface was covered with secondary antibody [Goat Anti-Rabbit IgG H \& L (HRP)] for 30 min (ART NO: ab97051-1 mg, Abcam Co.) and finally placed on the diamino benzene hydrochloride solution containing $0.03 \% \mathrm{H}_{2} \mathrm{O}_{2}$ as chromogen for peroxidase activity for 10-15 min. It was then placed in Harris hematoxylin for $1 \mathrm{~min}$ as counterstain.

Breast cancer tissue was considered for positive control. Gingival tissue was considered for negative control.

\section{Statistical Analysis}

All slides were evaluated with an optical microscope (Olympus BX41, Olympus, Tokyo, Japan) with a magnification of 40 times by two independent pathologists who were unaware of the clinical features of the samples. Immunohistochemical expression evaluation of SIRT-1 was done so that the five randomly selected histology fields were considered and cell percentage of cytoplasm and nucleus condition was determined with a magnification of 400 in each sample obtaining a score ${ }^{1}$ as follows:

Score 0 : tumors where $0-5 \%$ of cancer cells were stained. Score 1: tumors where $6-25 \%$ of cancer cells were stained. Score 2: tumors where $26-50 \%$ of cancer cells were stained. Score 3: tumors where $51-75 \%$ of cancer cells were stained. Score 4: tumors where $76-100 \%$ of cancer cells were stained.

Cytoplasm and nucleus staining intensity of epithelial tumor cells stained with SIRT-1 was recorded in four different degrees including: no staining (0), weak staining (1), moderate staining (2) and severe staining (3). Finally, based on the total staining intensity and percentage of stained cells, the final score was computed as follows: Negative (from 0 to 2) and positive (from 3 to 7 ).

Statistical analysis was performed using SPSS statistical software. Immunohistochemical staining intensity and percentage differences of staining in each group was determined by Chi-square test and Mann-Whitney test was done to examine the relationship between clinical factors of staining intensity and percentage of staining.

\section{Results}

About 60 samples, including 30 paraffin blocks of OSCC from $11(36.7 \%)$ men and $19(63.3 \%)$ women with a mean \pm SD age $68.70 \pm 15.55$ years and 30 normal tissue samples of patients referred for crown lengthening surgery were included. The mean \pm SD age of the patients in the healthy group was 39.20 \pm 12.42 years.

Among the OSCC specimens, 15 (50\%) had grade I, 12 (40\%) grade II and three (10\%) grade III OSCC. Moreover, in this group, buccal mucosa, gums, Rethro-molorpad, and lower part of the mouth were involved in 12 (40\%), 10 (33.3\%), six (20\%), and two $(6.7 \%)$ patients in this group.

In this study, SIRT1 staining both in the nucleus and in the cytoplasm of squamous cells was evaluated. The frequency distribution of the percentage of stained cells in terms of nuclear and cytoplasmic staining intensity in OSCC and normal oral mucosa is shown in Table 1.

SIRT1 expression was significantly seen in the cytoplasm and nucleus of tumor cells showing diffuse distribution in island of OSCC.

In terms of the percentage of stained cells in the core, 25 (83.3\%) cases of OSCC had score 4 (76-100\%) and in the cytoplasm, 28 (93.3\%) cases had score 4 (76-100\%), respectively.

In terms of intensity of nuclear staining in the cells of OSCCs, 18 (60\%) cases showed severe staining cytoplasm and in terms of staining intensity, 22 (73.3\%) cases showed weak staining.

According to the results, the mean \pm SD final scores of core staining in OSCC cells and normal oral mucosa were 6.07 \pm 1.780 and $3.00 \pm 1.597$, respectively. $69.9 \%$ of normal mucosa in the mouth and $93.4 \%$ of OSCC cells expressed a positive marker of SIRT1 (score 3 and above) and there was a significant difference in scores between the core staining in OSCC and oral mucosa $(P<0.001$; Table 2$)$.

We found that the mean \pm SD final scores of cytoplasm staining in OSCC cells and normal oral mucosa were $5.10 \pm$ 0.845 and $3.03 \pm 2.076$, respectively. About $53.4 \%$ of normal mucosa in the mouth and $96.7 \%$ of OSCC cells express a positive marker of SIRT1 (score 3 and above) and there was a significant difference in final scores between the OSCC and normal oral mucosa between the core and the oral mucosa oral squamous cell carcinoma $(P<0.001$; Fig. 1 and Table 3$)$.

Table 4 shows the immunoreactivity of OSCC in the core and cytoplasm. As shown, SIRT1 was expressed significantly in the core and cytoplasm of OSCC cells.

Comparing the SIRT1 expression in the two groups using Mann-Whitney test, we found a significant relationship between all the colors of SIRT1 in normal mucosa and oral squamous cell carcinoma in the percentage of cells stained in terms of core $(P=0.001)$, cytoplasm $(P=0.001)$, nuclear staining intensity $(P=0.001)$ and cytoplasm staining intensity $(P=0.001)$.

To examine the relationship between sex and age, clinical tumor stage, location of tumor, distant metastasis and metastasis to lymph nodes and smoking and crying, Mann-Whitney test was used for SIRT1 expression. ${ }^{1}$ We found no significant relationship between the rate and severity of core and cytoplasmic staining of tumor cells in OSCC and samples with clinicopathological factors, such as no lymph node metastasis and distant metastasis, smoking, age, sex, tumor location, 


\begin{tabular}{|c|c|c|c|c|}
\hline Expression & Grading & $\begin{array}{l}\text { Oral squamous } \\
\text { cell carcinoma (\%) }\end{array}$ & $\begin{array}{l}\text { Normal oral } \\
\text { mucosa }(\%)\end{array}$ & $P$-value \\
\hline \multirow{5}{*}{$\begin{array}{l}\text { Percentage of stained cells in } \\
\text { terms of nucleus }\end{array}$} & $<5$ & $2(6.7)$ & $6(20.0)$ & \multirow[t]{5}{*}{0.001} \\
\hline & $6-25$ & $0(0)$ & $11(36.7)$ & \\
\hline & $26-50$ & $0(0)$ & $9(30.0)$ & \\
\hline & $51-75$ & $3(10.0)$ & $3(10.0)$ & \\
\hline & $76-100$ & $25(83.3)$ & $1(3.3)$ & \\
\hline \multirow[t]{4}{*}{ Nuclear staining intensity } & No staining & $2(6.7)$ & $3(10.0)$ & \multirow[t]{4}{*}{0.001} \\
\hline & Weak & $1(3.3)$ & $7(23.3)$ & \\
\hline & Moderate & $9(30.0)$ & $19(63.3)$ & \\
\hline & Severe & $18(60.0)$ & $1(3.3)$ & \\
\hline \multirow{5}{*}{$\begin{array}{l}\text { Percentage of stained cells in } \\
\text { terms of cytoplasm }\end{array}$} & $<5$ & $0(0)$ & $7(23.3)$ & \multirow[t]{5}{*}{0.001} \\
\hline & $6-25$ & $1(3.3)$ & $7(23.3)$ & \\
\hline & $26-50$ & $1(3.3)$ & $2(6.7)$ & \\
\hline & $51-75$ & $0(0)$ & $88(26.7)$ & \\
\hline & $76-100$ & $28(93.3)$ & $6(20.0)$ & \\
\hline \multirow{4}{*}{$\begin{array}{l}\text { Cytoplasmic staining } \\
\text { intensity }\end{array}$} & No staining & $0(0)$ & $7(23.3)$ & \multirow[t]{4}{*}{0.016} \\
\hline & Weak & $22(73.3)$ & $15(50.0)$ & \\
\hline & Moderate & $8(26.7)$ & $8(26.7)$ & \\
\hline & Severe & $0(0)$ & $0(0)$ & \\
\hline
\end{tabular}

Table 2. Frequency distribution of the final core staining of cells in oral squamous cell carcinoma and normal oral mucosa

\begin{tabular}{llcccc}
\hline Expression & Group & Grading & Frequency & Percentage (\%) & Result \\
\hline Final nuclear & Oral squamous cell & 0 & 2 & 6.7 & - \\
staining scores & carcinoma & 1 & 0 & 0 & - \\
& & 2 & 0 & 0 & - \\
& 3 & 0 & 0 & + \\
& 4 & 0 & 0 & + \\
& & 5 & 3 & 10.0 & + \\
& & 6 & 8 & 26.7 & + \\
& & 7 & 17 & 56.7 & + \\
& & 0 & 3 & 10 & - \\
& 1 & 2 & 6.7 & - \\
& 2 & 4 & 13.3 & - \\
& 3 & 10 & 33.3 & + \\
& 4 & 7 & 23.3 & + \\
& & 5 & 3 & 10.0 & + \\
& 6 & 0 & 0 & + \\
& & 7 & 1 & 3.3 & +
\end{tabular}



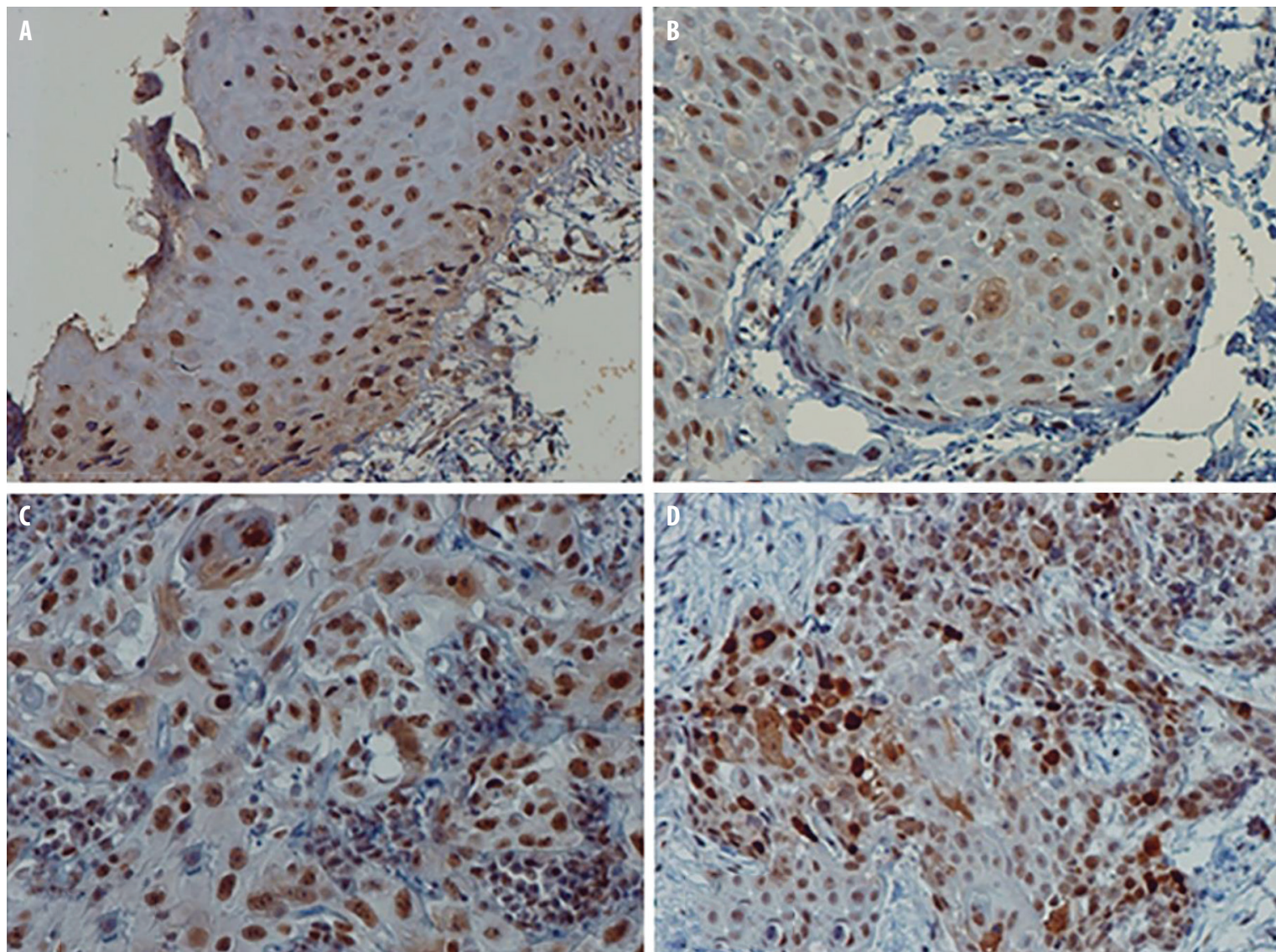

Fig. 1 Immunostaining of SIRT1 in (A) normal oral mucosa, (B) well-differentiated oral squamous cell carcinoma, (C) moderately differentiated oral squamous cell carcinoma and (D) poorly differentiated oral squamous cell carcinoma (magnification: 400X).

\begin{tabular}{|c|c|c|c|c|c|}
\hline Expression & Group & Grading & Frequency & Percentage (\%) & Result \\
\hline \multirow{16}{*}{$\begin{array}{l}\text { Final } \\
\text { cytoplasmic } \\
\text { staining scores }\end{array}$} & \multirow{8}{*}{$\begin{array}{l}\text { Oral squamous } \\
\text { cell carcinoma }\end{array}$} & 0 & 0 & 0 & - \\
\hline & & 1 & 0 & 0 & - \\
\hline & & 2 & 1 & 3.3 & - \\
\hline & & 3 & 1 & 3.3 & + \\
\hline & & 4 & 0 & 0 & + \\
\hline & & 5 & 20 & 66.7 & + \\
\hline & & 6 & 8 & 26.7 & + \\
\hline & & 7 & 0 & 0 & + \\
\hline & \multirow{8}{*}{$\begin{array}{l}\text { Normal oral } \\
\text { mucosa }\end{array}$} & 0 & 6 & 20.0 & - \\
\hline & & 1 & 0 & 0 & - \\
\hline & & 2 & 8 & 26.7 & - \\
\hline & & 3 & 2 & 6.7 & + \\
\hline & & 4 & 6 & 20.0 & + \\
\hline & & 5 & 3 & 10.0 & + \\
\hline & & 6 & 5 & 16.7 & + \\
\hline & & 7 & 0 & 0 & + \\
\hline
\end{tabular}


Table 4. Immunoreactivity of cells (SIRT1) in the core and cytoplasm of cells in oral squamous carcinoma cells

\begin{tabular}{llcc}
\hline Location & Immunoactivity & Abundance (\%) & P-value \\
\hline Core & Negative & $2(6.7)$ & 0.021 \\
& Positive & $28(93.3)$ & \\
Cytoplasm & Negative & $1(3.3)$ & 0.001 \\
& Positive & $29(96.7)$ & \\
\hline
\end{tabular}

tumor size and tumor clinical stage $(P>0.05)$. However, in the final check, there was a significant relationship between OSCC samples and normal tissue in the mouth with respect to the final scores of the cytoplasmic and nuclear core $(P<0.001)$.

\section{Discussion}

The results of this study suggest the higher expression of SIRT1 in OSCC compared with normal oral mucosa, which confirms the role of SIRT1 in carcinogenesis.

Oral squamous cell carcinoma is related to genetic changes with lack of cell growth and differentiation. ${ }^{1}$ Tumorigenesis is the result of a series of genetic changes leading to abnormal activity of oncogenes and inactivation of tumor suppressors. ${ }^{2}$ Molecular markers can play an indispensable role in predicting tumor aggressiveness. By identifying the biological markers, we can improve clinical staging system's ability to increase and predict prognosis and progression of OSCC.

Jung et al. ${ }^{13}$ reported that SIRT1 promotes tumor by P53 inhibition. They found that SIRT1 expression is a good prognostic factor for colorectal cancer, and in this regard, is intersected with $\beta$-catenin more than P53. Also SIRT1 reduced protein expression of BCL2.

Moreover, in our study, the SIRT1 expression significantly observed in the nucleus and cytoplasm of tumor cells is shown in diffuse distribution at oral squamous cell carcinoma neoplastic island. Its expression in OSCC was higher than normal oral mucosa that is consistent with studies on colon cancer, ${ }^{14}$ squamous cell carcinoma, ${ }^{15}$ and basal cell carcinoma and Bowen's disease $\mathrm{e}^{16}$ showing overexpression of SIRT1 in cancer tissue compared to normal tissue.

Michishita et al. ${ }^{16}$ also observed that the staining SIRT1 in normal tissue and dysplastic and cancerous tissue is mainly appeared in the core in a small number of samples, all of which has cytoplasmic expression. In another study, SIRT1 had more gene expression in OSCC than the rest of the family. ${ }^{17}$

Byles et al. ${ }^{18}$ also used human tissue and reported that SIRT1 is focused mainly in the core of normal cells, but is apparently seen deformed in the cytoplasm of cancer cells, which is consistent with the results of this study.

In this study, core and cytoplasmic expression of SIRT1 was not significantly associated with any clinicopathological factors. Another study showed that SIRT1 expression level was not related to depth of invasion, differentiation and tumor size, type of tumor, lymph node metastasis, and the patient's age. ${ }^{19}$ Another study also showed a significant relationship between the expression of SIRT1 and distant metastasis. ${ }^{20}$ SIRT1 participation in other cancers have been reported including breast, ${ }^{20}$ colorectal, ${ }^{13}$ lung, ${ }^{21}$ and skin ${ }^{16}$ cancers and Bowen's disease. ${ }^{16}$

We found no significant relationship among the percentage of core and cytoplasmic staining of tumor cells in a sample of oral squamous cell carcinoma with tumor grade. Inconsistently, Lin and Peng ${ }^{21}$ found that there was a significant relationship between the survival of SIRT1 and average and weak of histological grade. Kabra et al. ${ }^{22}$ also showed that in stage I, II, and III colorectal adenocarcinoma SIRT1 expression significantly increases. However, in our study there is no link between SIRT1 expression and stage of the disease. SIRT1 expression plays an important role in predicting oral squamous carcinomas, ${ }^{13}$ whereas in our study SIRT 1 had no relationship with prognostic factors.

Due to the significant difference in the expression of SIRT1, we found a significant relationship between OSCC and normal oral mucosa with respect to SIRT1 expression indicating that it can be a possible prognostic marker. Therefore, by further studies in this regard and studying the role of this protein, it could be used as a therapeutic target molecule in the treatment of patients with OSCC.

\section{Conclusion}

Overexpression of SIRT1 in oral squamous cell carcinoma samples compared with normal tissue of the mouth indicates its role in carcinogenesis. With further studies of SIRT1 in this regard, SIRT1 may be used to treat the target molecule.

\section{Conflicts of Interest}

The authors declare that they have no conflicts of interest.

\section{References}

1. Yu C, Chen K, Zheng H, Guo X, Jia W, Li M, et al. Overexpression of astrocyte elevated gene-1 (AEG-1) is associated with esophageal squamous cell carcinoma (ESCC) progression and pathogenesis. Carcinogenesis. 2009:30:894-901.

2. Noch EK, Khalili K. The role of AEG-1/MTDH/LYRIC in the pathogenesis of central nervous system disease. Adv Cancer Res. 2013;120:159-192.

3. Noguchi A, Li X, Kubota A, Kikuchi K, Kameda Y, Zheng H, et al. SIRT1 expression is associated with good prognosis for head and neck squamous cell carcinoma patients. Oral Surg Oral Med Oral Pathol Oral Radiol. 2013;115:385-392.

4. Seyedmajidi M, Shafaee S, Siadati S, Khorasani M, Bijani A, Ghasemi N. Cyclo-oxygenase-2 expression in oral squamous cell carcinoma. J Cancer Res Ther. 2014;10:1024-1029.

5. Shakib PA, Ensani F, Abdirad A, Valizadeh B, Seyedmajidi M, Sum S. CD44 and CD74: the promising candidates for molecular targeted therapy in oral squamous cell carcinoma. Dent Res J (Isfahan). 2015;12:181-186.

6. Emdad L, Das SK, Dasgupta S, Hu B, Sarkar D, Fisher PB. AEG-1/MTDH/LYRIC: signaling pathways, downstream genes, interacting proteins, and regulation of tumor angiogenesis. Adv Cancer Res. 2013;120:75-111.

7. Longo VD, Kennedy BK. Sirtuins in aging and age-related disease. Cell. 2006:126:257-268.

8. Murayama A, Ohmori K, Fujimura A, Minami H, Yasuzawa-Tanaka K, Kuroda T, et al. Epigenetic control of rDNA loci in response to intracellular energy status. Cell. 2008;133:627-639.

9. Bosch-Presegué L, Vaquero A. The dual role of sirtuins in cancer. Genes. Cancer. 2011;2:648-662.

10. Liu T, Liu PY, Marshall GM. The critical role of the class III histone deacetylase SIRT1 in cancer. Cancer Res. 2009:69:1702-1705.

11. van der Schroeff MP, van Schie K, Langeveld TP, Looman C, Baatenburg de Jong RJ. Model-assisted predictions on prognosis in HNSCC: do we learn? Eur Arch Otorhinolaryngol. 2010;267:1445-1448.

12. Emdad L, Sarkar D, Su ZZ, Lee SG, Kang DC, Bruce JN, et al. Astrocyte elevated gene-1: recent insights into a novel gene involved in tumor progression, metastasis and neurodegeneration. Pharmacol Ther. 2007;114:155-170.

13. Jung W, Hong KD, Jung WY, Lee E, Shin BK, Kim HK, et al. SIRT1 expression is associated with good prognosis in colorectal cancer. Korean J Pathol. 2013:47:332-339. 
14. Jin Q, Yan T, Ge X, Sun C, Shi X, Zhai Q. Cytoplasm-localized SIRT1 enhances apoptosis. J Cell Physiol. 2007:213:88-97.

15. Hida Y, Kubo Y, Murao K, Arase S. Strong expression of a longevity-related protein, SIRT1, in Bowen's disease. Arch Dermatol Res. 2007;299:103-106.

16. Michishita E, Park JY, Burneskis JM, Barrett JC, Horikawa I. Evolutionarily conserved and nonconserved cellular localizations and functions of human SIRT proteins. Mol Biol Cell. 2005;16:4623-4635.

17. Lai CC, Lin PM, Lin SF, Hsu CH, Lin HC, Hu ML, et al. Altered expression of SIRT gene family in head and neck squamous cell carcinoma. Tumour Biol. 2013;34:1847-1854.

18. Byles V, Chmilewski LK, Wang J, Zhu L, Forman LW, Faller DV, et al. Aberrant cytoplasm localization and protein stability of SIRT1 is regulated by PI3K/ IGF-1R signaling in human cancer cells. Int J Biol Sci. 2010;6:599-612.
19. Yu DF, Jiang SJ, Pan ZP, Cheng WD, Zhang WJ, Yao XK, et al. Expression and clinical significance of Sirt1 in colorectal cancer. Oncol Lett. 2016;11: $1167-1172$.

20. Lee H, Kim KR, Noh SJ, Park HS, Kwon KS, Park BH, et al. Expression of DBC and SIRT1 is associated with poor prognosis for breast carcinoma. Hum Pathol. 2011:42:204-213.

21. Lin SY, Peng F. Association of SIRT1 and HMGA1 expression in non-small cell lung cancer. Oncol Lett. 2016;11:782-788.

22. Kabra N, Li Z, Chen L, Li B, Zhang X, Wang C, et al. SirT1 is an inhibitor of proliferation and tumor formation in colon cancer. J Biol Chem. 2009:284:18210-18217.

This work is licensed under a Creative Commons Attribution-NonCommercial 3.0 Unported License which allows users to read, copy, distribute and make derivative works for non-commercial purposes from the material, as long as the author of the original work is cited properly. 